\title{
Evidence-Based Psychosocial Treatments for Pediatric Mood and Anxiety Disorders
}

\author{
Heather A. MacPherson, $M A^{1, *}$ \\ Mary A. Fristad, PhD, $A B P P^{1,2}$
}

\author{
Address \\ ${ }^{*}, 1$ Departments of Psychiatry and Psychology, The Ohio State University, 1670 \\ Upham Drive, Suite 460, Columbus, $\mathrm{OH}$ 43210-1250, USA \\ Email: heather.macpherson@osumc.edu \\ ${ }^{2}$ Departments of Psychiatry, Psychology, and Nutrition, The Ohio State University, \\ Columbus, OH, USA \\ Email:mary.fristad@osumc.edu
}

Published online: 20 December 2013

(C) Springer International Publishing AG 2013

\begin{abstract}
Keywords Depression - Bipolar disorder - Mania - Mood - Anxiety - Separation anxiety disorder - Generalized anxiety disorder - Social anxiety disorder - Panic disorder - Specific phobia - Obsessive-compulsive disorder . Acute/posttraumatic stress disorders - Cognitive-behavioral therapy - Interpersonal therapy - Attachment-based family therapy - Parent-child interaction therapy - Family psychoeducation - Dialectical behavior therapy . Interpersonal and social rhythm therapy - Healthy lifestyle - Social effectiveness therapy - Attention bias modification training - Panic control treatment - Trauma-focused cognitive-behavioral therapy - Eye movement desensitization and reprocessing
\end{abstract}

\section{Opinion statement}

Treatment of internalizing disorders in youth often entails a multimodal approach, including pharmacotherapy, psychotherapy, and systems-based intervention in familial, school, and social settings. Prior to selecting appropriate treatments, a comprehensive, lifetime assessment employing multiple informants (youth, parents, teachers) and methods (questionnaires, semi-structured evaluation, clinical interview) should be conducted. Given the high rate of co-occurrence of multiple internalizing disorders, comorbidity of mood and anxiety with other psychiatric problems, and symptom overlap across diagnostic categories, thorough assessment taking into account differential diagnoses is essential. Following evaluation, treatment selection should be based on the primary presenting problem, degree of impairment, and current evidence. For moderate-to-severe adolescent depression, a combination of cognitive-behavioral therapy (CBT) with pharmacotherapy (e.g., selective serotonin reuptake inhibitors [SSRIs]) has the strongest empirical base. Mounting evidence also supports the use of CBT and interpersonal therapy (IPT) as monotherapies for depressed youth. CBT for depression involves identifying and challenging cognitive distortions, behavioral activation, problem solving, and emotion regulation, while IPT aims to reduce interpersonal con- 
flict via interpersonal problem-solving and communication skills. For pediatric bipolar disorders, family psychoeducation about the etiology, course, and treatment of mood disorders, plus skill building (problem solving, CBT, communication, emotion regulation), should be employed adjunctive to pharmacotherapy. Finally, for anxiety disorders, various forms of CBT have demonstrated efficacy. Typically, use of SSRIs concurrently with CBT offers additional benefit for anxious youth. Though the format and focus of CBT varies depending on the specific anxiety disorder, most interventions involve psychoeducation, emotion identification and management strategies, cognitive restructuring, exposure, and familial involvement. Recent preliminary research also supports the use of other psychotherapies (e.g., attachment-based family therapy, parent-child interaction therapy, attention bias modification training) and healthy lifestyle changes (e.g., sleep, diet, exercise). In conclusion, CBT-based treatments currently have the most empirical support, and should be considered first-line psychosocial interventions for pediatric internalizing disorders.

\section{Introduction}

Though previously believed to be disorders of adulthood, research suggests that pediatric mood and anxiety disorders are common and of significant public health concern. Depressive disorders (major depressive, disruptive mood dysregulation, and premenstrual dysphoric disorders; persistent and/or other specified/ unspecified depressive disorders) are characterized by dysregulated mood (dysphoria/irritability) and/or anhedonia, vegetative changes (fatigue, sleep difficulties), and cognitive disturbances (feelings of worthlessness/ guilt, thoughts of death/suicide). Though bipolar disorders (bipolar I, II, and cyclothymic disorders; other specified/unspecified bipolar and related disorders) often include depressive episodes, their hallmark lies in manic symptoms (elated and/or irritable mood, pressured speech, grandiosity, decreased need for sleep, risky behavior). Pediatric anxiety is comprised of a larger class of disorders (separation anxiety, generalized anxiety, social anxiety, and panic disorders; selective mutism; specific phobia; obsessive-compulsive disorder; acute/ posttraumatic stress disorders), though all are characterized by: fear, anxiety, and/or worry; physiological arousal; and avoidance (or behaviors aimed at prevention) of feared stimuli/outcomes. See the Diagnostic and Statistical Manual of Mental Disorders, $5^{\text {th }}$ edition for diagnostic criteria $[1 \bullet \bullet]$.

Internalizing disorders in youth occur frequently and have high rates of comorbidity. A recent epidemiological study with adolescents reported prevalence of $31.9 \%$ for any anxiety disorder and $14.3 \%$ for any mood disorder ( $11.7 \%$ for depressive disorders; $2.9 \%$ for bipolar I and II disorders) [2]. For school-aged children, anxiety disorders affect $10-20 \%$ of youth [3], while depression and bipolar disorders each impact $2 \%$ of children $[4,5 \bullet]$. Though comorbidity is the rule, rather than the exception, when it comes to pediatric psychiatric disorders, co-occurrence of mood and anxiety is particularly common $[2,4]$. Development of internalizing disorders typically involves interaction between biological vulnerability and environmental stressors. Symptoms follow a waxing and waning course and cause considerable social, academic, and familial impairment.

Regarding intervention, pharmacotherapy is common in treating youth internalizing disorders. However, psychotherapy is vital for teaching coping skills and addressing psychosocial stressors, while healthy lifestyle changes can regulate nutritional/circadian rhythm imbalances. For depression, cognitive-behavioral therapy (CBT) and interpersonal therapy are well established, while attachment-based family therapy and parent-child interaction therapy show promise. Family psychoeducation plus skill building is recommended for bipolar disorders, though CBT, dialectical behavior therapy, and interpersonal and social rhythm therapy have mounting evidence. Healthy lifestyle changes (sleep, diet, exercise) are commonly emphasized in mood disorder treatments. Regarding anxiety, varying formats of CBT are efficacious for separation anxiety, generalized anxiety, and social anxiety disorders, selective mutism, and specific phobia; however, attention bias modification training is also impactful for anxious 
youth. Regarding disorder-specific interventions, research supports the use of: social effectiveness therapy for social anxiety; panic control treatment for panic disorder; CBT incorporating exposure/response prevention for obsessive-compulsive disorder; and trauma-focused CBT for acute/posttraumatic stress disorders. Below we offer a summary of interventions and evidence classification based on recent re- search (emphasizing randomized controlled trials [RCTs] and studies of youth with diagnosable internalizing disorders). The Journal of Clinical Child and Adolescent Psychology offers in-depth review of psychosocial treatment outcome studies for depression

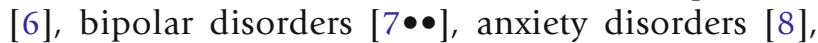

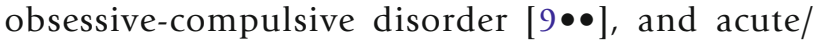
posttraumatic stress disorders [10].

\title{
Psychosocial treatments
}

\section{Depressive disorders}

Cognitive-behavioral therapy

\author{
Overview
}

Empirical support

- $\quad$ CBT teaches cognitive restructuring (identifying and challenging negative thoughts/distortions), behavioral activation, problem solving skills, and emotion regulation strategies.

- This approach may be particularly effective for youth with high levels of cognitive distortion and inactivity.

- CBT has the most empirical support for treatment of adolescent depression, particularly when paired with pharmacotherapy (usually selective serotonin reuptake inhibitors [SSRIs]) [Class I]. For example, in the Treatment of Adolescent Depression Study (TADS), where adolescents were randomized to individual CBT (with parental component), fluoxetine, CBT+fluoxetine, or pill-placebo, combined treatment was associated with the most rapid and complete response $[11,12]$. Similarly, in the Treatment of Resistant Depression in Adolescents study (TORDIA), switching antidepressants and adding individual CBT (with parental component) was superior to a medication switch alone [13]. However, according to other RCTs, combined treatment may not offer increased benefit over CBT monotherapy [14] or pharmacotherapy alone [14, 15]. Though the advantage of combined treatment over monotherapy seems to dissipate over time, these reasons are unclear (e.g., uncontrolled treatment usage during follow-up), and shortcomings of studies (e.g., attrition) limit interpretability of this finding.

- Research also supports the use of CBT as a monotherapy in treatment of adolescent depression [Class I]. Numerous RCTs employing various CBT formats (group, individual, included parental component) and control conditions (waitlist; supportive, family, and relaxation therapies; life skills tutoring) demonstrated significant improvement in depressive symptoms, though be- 
tween-group differences were not always maintained at follow-up assessments [e.g., 16-19]. One effectiveness study including children and adolescents using a usual clinical care control found significant improvement in depressive symptoms among both groups, though CBT was superior in terms of alliance, cost, length of treatment, and use of other services [20]. Finally, TADS monotherapy outcomes indicated that CBT did not differ from placebo and performed less well than fluoxetine in acute treatment, but monotherapy outcomes converged over time [11, 12].

- Most psychosocial treatment outcome studies for childhood depression included samples of at-risk youth with elevated symptoms [6]. Though no RCTs focused exclusively on school-aged children with a diagnosable disorder, several RCTs with mixed age groups demonstrated efficacy of CBT for depressed youth [e.g., 19-22, Class I]. In addition, two open trials of CBT-based treatments for school-aged children with diagnosed depression reported promising results $[23,24]$. One effectiveness RCT using a mixed sample of children with depression, anxiety, and/or conduct problems demonstrated superiority of a modular intervention (flexible application of CBT) over usual care and standard evidence-based treatments (separate, disorder-specific

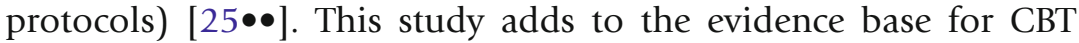
in treatment of youth depression, and also suggests that a flexible approach may be more effective in practice settings.

Interpersonal therapy

\section{Overview}

- Interpersonal therapy (IPT) aims to decrease interpersonal conflicts by teaching interpersonal problem-solving and communication skills. Targeted problem areas include grief, role transitions, role disputes, and interpersonal deficits.

- This approach may be particularly effective for youth with conflictual familial and peer relationships, which often exacerbate depressive symptoms.

\section{Empirical support}

- Four RCTs demonstrated the efficacy of IPT in treatment of adolescent depression [Class I]. Two studies found that individual IPT was superior to treatment as usual (TAU) and clinician monitoring in ameliorating depressive symptoms [26, 27]. RCTs of CBT versus IPT showed significant improvement in depressive symptoms overall, but mixed results regarding superiority of intervention $[28,29]$.

- As with CBT, most studies of IPT used adolescent samples, though one open trial examining IPT with depressed, school-aged children showed promise, further supporting the use of IPT for depression across development [30, Class IV]. 
Attachment-based family therapy

Overview

- Attachment-based family therapy (ABFT) aims to strengthen and/or rebuild secure parent-child relationships by targeting: parental criticism/hostility, distress, skills, and disengagement; and adolescent mo-

Empirical support tivation, negative self-concept, affect regulation, and disengagement.

- Though examined less rigorously than CBT and IPT, two small-scale RCTs of ABFT with adolescents using a waitlist control (WLC) and TAU comparisons demonstrated significant reduction in depressive symptoms [31, 32, Class II].

- One RCT with children investigated a similar family-based treatment (systems integrative family therapy) versus focused individual psychodynamic psychotherapy, and also reported positive preliminary results [33, Class III].

Parent-child interaction therapy - emotion development

Overview

Empirical support

- Parent-child interaction therapy - emotion development (PCIT-ED) was developed for depressed preschoolers and involves strengthening the parent-child relationship via teaching and in vivo coaching of: positive play techniques; effective commands and punishment; and emotional competence and regulation.

- One RCT using a psychoeducation control demonstrated positive, significant results in both groups, though PCIT-ED showed significance in a greater number of domains. PCIT-ED was also superior for executive functioning and emotion recognition skills [34•, Class III].

Healthy diet and lifestyle

\section{Overview}

- Eating balanced meals, maintaining a regular sleep cycle, and engaging in physical exercise can also alleviate depressive symptoms in youth by regulating nutritional, metabolic, and circadian rhythm functioning, and also by improving self-esteem and self-cognitions.

Empirical support

- While diet and sleep interventions have not been studied in isolation, many of the aforementioned psychotherapies emphasize healthy lifestyle changes in treatment. In addition, aerobic exercise has shown promising preliminary results in an open study with depressed adolescents [35, Class IV], and is commonly targeted in psychosocial treatment of depression. 


\section{Bipolar disorders}

Family psychoeducation plus skill building

Overview

\section{Empirical support}

- Family psychoeducation teaches families about mood symptoms, course, and effective treatment, while skill building involves symptom management strategies (e.g., CBT, problem solving, communication, emotion regulation).

- This intervention has the most empirical support in treatment of pediatric bipolar disorders [Class II]. It has been examined adjunctive to TAU and/or pharmacotherapy with both children and adolescents in individual-family and multiple-family formats in three large RCTs: multi-family psychoeducational psychotherapy+TAU versus WLC+ TAU; family-focused treatment (FFT) + pharmacotherapy versus enhanced care control+pharmacotherapy; and FFT for youth at highrisk for developing bipolar disorders + pharmacotherapy versus family education control+pharmacotherapy. Results demonstrated significant improvement in mood and behavior symptoms following treatment and over time $[36,37,38 \bullet \bullet, 39 \bullet]$.

Cognitive-behavioral therapy

\section{Overview}

- CBT-based interventions for pediatric bipolar disorders are very similar to aforementioned family psychoeducation plus skill building treatments, differing mostly in their level of empirical support.

\section{Empirical support}

- CBT+pharmacotherapy has been evaluated with children and adolescents in individual-family and multiple-family formats, as well as in an adolescent-only format (with limited familial involvement). Two open trials and one study with matched historical controls demonstrated improvement in mood and some comorbid symptoms following treatment [40-42, Class III].

Dialectical behavior therapy

\section{Overview}

- Dialectical behavior therapy (DBT) involves individual therapy, family skills training, and as-needed telephone coaching to target problematic behaviors and replace them with effective coping skills (i.e., mindfulness, distress tolerance, emotion regulation, interpersonal effectiveness, walking the middle path).

- This intervention may be particularly helpful for youth who exhibit suicidal thoughts/behaviors and/or non-suicidal self-injury. 


\section{Empirical support}

- Individual-family DBT+pharmacotherapy was evaluated in one published open trial with adolescents and demonstrated significant improvement in depression, emotion dysregulation, and suicidality [43, Class IV].

Interpersonal and social rhythm therapy

\section{Overview}

\section{Empirical support}

Healthy diet and lifestyle

\section{Overview}

\section{Empirical support}

- Though diet, sleep, and exercise interventions have not been examined as monotherapies, many of the aforementioned psychosocial interventions specifically target these healthy lifestyle changes in treatment.

\section{Separation anxiety, generalized anxiety, and social anxiety disorders}

Separation anxiety, generalized anxiety, and social anxiety are the most common anxiety disorders and frequently comorbid. Thus, they are often studied simultaneously in outcome trials and are reviewed together here. While selective mutism is also a common childhood anxiety disorder, no RCTs have exclusively examined treatment of this condition. However, youth with selective mutism have been included in several of the studies reviewed below.

Cognitive-behavioral therapy

\section{Overview}

- In general, CBT for anxiety disorders targets physiologic, cognitive, and behavioral symptoms via psychoeducation, emotion identification, somatic management strategies, cognitive restructuring, exposure, familial involvement, and relapse prevention. 


\section{Empirical support}

- CBT for youth with separation anxiety disorder emphasizes identification of anxious, separation-related thoughts and feelings, recognition of physical arousal, and development of more effective coping skills (cognitive restructuring, relaxation strategies).

- Key elements of CBT for generalized anxiety disorder involve identifying and challenging irrational fears and worries, and practicing exposure to feared outcomes via role plays, imagery, and in vivo exercises.

- Important features of CBT for social anxiety disorder include cognitive restructuring of irrational thoughts related to social settings and exposure to avoided social situations.

- CBT has the most empirical support for treatment of youth anxiety disorders [Class I]. Similar to the adolescent depression literature, combination of CBT+pharmacotherapy (usually SSRIs) offers added benefit for anxious youth. For example, the Child/ Adolescent Anxiety Multimodal Study (CAMS) found that all active treatments (individual CBT with parental component, sertraline, CBT+sertraline) were superior to pill-placebo, though combined treatment was superior to either monotherapy alone [45].

- CBT monotherapy has also demonstrated efficacy in improving anxiety symptoms in numerous RCTs with both children and adolescents [Class I]. These studies provide support for several formats of CBT (individual, group, included parental component, parent group only) in the treatment of pediatric anxiety disorders when compared with WLC, family-based education support, and group support+attention control [e.g., 46, 47•, 48-50, 51•].

Though an effectiveness trial using usual care control did not demonstrate significant between-group differences, clinical improvements across groups were evident, and youth receiving CBT used fewer additional services [52].

- In addition, while a variety of CBT formats have demonstrated efficacy in treatment of pediatric anxiety disorders, group CBT is particularly effective for social anxiety disorder compared to WLC or attention control [53, 54, Class II].

- Finally, CBT with computerized components may ameliorate anxious symptoms to a similar degree as clinic-delivered CBT $[55,56 \bullet$, 57, Class II]. For example, recent studies demonstrated superiority of computerized and/or computer-assisted CBT over WLC and a computer-assisted education, support, and attention control. In addition, CBT with computerized components produced similar, comparable outcomes as clinic-based CBT. These studies further support use of CBT with anxious youth and also offer novel delivery formats to aid in transportability and dissemination of this treatment to real-world settings. 
Social effectiveness therapy

Overview

Empirical support

- Social effectiveness therapy (SET) is a behavioral group treatment specifically for social anxiety disorder involving psychoeducation, social skills training, peer generalization experiences, and in vivo exposure.

- SET was effective in ameliorating childhood anxiety symptoms and improving social skills in an RCT using a study skills control [58]. SET also demonstrated significantly greater treatment response rates relative to fluoxetine and placebo, and greater improvements in social skills, among a sample including children and adolescents [59, Class II].

Attention bias modification training

Overview

Empirical support

- Attention bias modification training (ABMT) for pediatric anxiety disorders is a novel treatment approach that teaches youth to shift their attention away from threat stimuli via computer training tasks.

- The first RCT of ABMT reported significant reduction in youths' anxiety symptoms compared with placebo attention training with threat and neutral stimuli $[60 \bullet \bullet]$. A second RCT examined a version of ABMT that trained attention towards positive stimuli (as opposed to solely away from threat) and demonstrated positive findings when compared with attention training control [61, Class II].

Parent-child interaction therapy

Overview

Empirical support

- Parent-child interaction therapy (PCIT) combines elements of play therapy and behavioral principles, and teaches parents positive attention, problem solving, and effective communication skills via in vivo training with children.

- One open trial PCIT for youth with varied anxiety disorders reported promising preliminary results [62, Class IV].

\section{Specific phobia}

Exposure

\section{Overview}

- This CBT-based treatment focuses on graded, hierarchical exposure to stimuli via imaginal and/or in vivo exercises. 


\section{Empirical support}

- Exposure-based interventions have been evaluated in RCTs using several sessions or one session compared with WLC or educational control, with results demonstrating decreases in anxiety [e.g., 63, 64, Class II].

\section{Panic disorder}

Panic control treatment

\section{Overview}

- Panic control treatment is a CBT-based intervention that involves psychoeducation, coping skills, physiologic symptom reduction, cognitive restructuring, interoceptive exposure, and relapse prevention.

\section{Empirical support}

- One RCT of panic control treatment demonstrated reduction of panic, anxiety, and depression when compared with self-monitoring and therapist check-in control [65, Class III].

\section{Obsessive-compulsive disorder}

Cognitive-behavioral therapy

\section{Overview}

- CBT for obsessive-compulsive disorder (OCD) includes psychoeducation, hierarchy building, exposure/response prevention, cognitive strategies, reward programs, family/parent training, and relapse prevention.

\section{Empirical support}

- Similar to research on psychosocial interventions for pediatric depression and anxiety, individual CBT is the most efficacious treatment for youth with OCD, particularly when combined with medication (e.g., SSRIs) [66, 67••, Class II]. The Pediatric OCD Treatment Study (POTS I) demonstrated superiority of CBT+sertraline over either monotherapy alone in terms of symptom reduction, though remission rates were comparable for combined treatment and CBT alone [66]. POTS II also demonstrated superiority of CBT+ pharmacotherapy over pharmacotherapy alone and pharmacotherapy+instructions in CBT [67••]. Two recent RCTs also found promising results when augmenting individual CBT with D-cycloserine, compared with individual CBT+placebo $[68 \bullet$, 69].

- Other formats of CBT may also be effective in treatment of pediatric OCD. For example, family-focused individual CBT demonstrated equivalent, positive results as compared to family-focused group CBT, and superiority over WLC and relaxation control [70-72, Class II]. 
- Group CBT also showed promise in several recent open trials [73, 74, Class III].

- Finally, CBT delivery via webcam is also a promising modality, according to one RCT using WLC [75, Class III]. This delivery format can again aid in widespread dissemination of CBT.

\section{Acute/posttraumatic stress disorders}

Trauma-focused cognitive-behavioral therapy

(and related interventions)

\section{Overview}

- Trauma-focused CBT (TF-CBT) for acute and posttraumatic stress disorders (PTSD) involves psychoeducation, relaxation and coping skills, affective modulation strategies, cognitive coping processing, trauma narrative development, in vivo and/or imaginal exposure to trauma reminders, and parental involvement.

\section{Empirical support}

- Numerous RCTs employing various control conditions (child-centered therapy, family therapy, routine clinical services, WLC) support the use of individual TF-CBT (commonly with parental involvement) in treatment

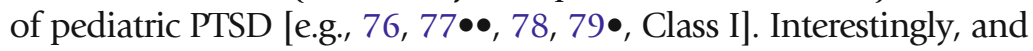
unlike aforementioned examinations of combined psychosocial + pharmacological interventions for internalizing disorders, addition of medication to TF-CBT (TF-CBT+sertraline versus TF-CBT+placebo) does not offer added benefit [80]. However, for youth exposed to a single trauma, an RCT comparing TF-CBT with and without exposure found comparable symptom improvement, suggesting that exposure may not be necessary for treatment gains among less impaired youth [81].

- Some of the treatments used as control conditions in the above-mentioned studies (child-centered therapy, family therapy) demonstrated some positive findings, though TF-CBT was typically superior [76, 78, Class III].

- Interventions similar to TF-CBT, which emphasize some treatment components more than others, also show promise. For example, CBT (focusing mainly on cognitive factors) resulted in significant improvement in anxiety symptoms and functioning compared to WLC [82, Class III]. Also, in a study of narrative exposure therapy (brief intervention focusing on development of a narrative encompassing all experienced traumas) versus meditation-relaxation, both treatments were effective in ameliorating PTSD symptoms [83, Class III].

School-based cognitive-behavioral therapy

\section{Overview}

- Though similar to TF-CBT, school-based CBT differs in the setting in which it occurs (school), and delivery format (group). The inter- 


\section{Empirical support}

Parent-child interaction therapy vention includes psychoeducation, graded exposures, cognitive and coping strategies, and social skills.

- One RCT reported comparable results for school-based CBT and individual TF-CBT [84]. A second study found support for schoolbased CBT+classroom psychoeducation/coping skills versus classroom psychoeducation/coping skills alone, though the active comparison condition also demonstrated some symptom reduction [85, Class II].

Eye movement desensitization and reprocessing

Empirical support

Overview

Overview

Empirical support
- Eye movement desensitization and reprocessing (EMDR) involves graduated imaginal exposure while the child simultaneously visually tracks therapist hand movements. This tracking procedure is hypothesized to aid in cognitive processing of the traumatic event during exposure.

- EMDR demonstrated nearly equivalent, positive results when compared with CBT [86]. A more recent RCT of CBT versus EMDR again showed significant symptom reduction in both groups, though treatment gains for EMDR were reached in fewer sessions [87, Class II].

\author{
Empirical support
}

- In two RCTs using WLC, PCIT has shown positive results among families at risk for or with a history of child maltreatment [88, 89•, Class II].

- Similar to PCIT for anxiety, PCIT for child maltreatment focuses on improving parent-child relations by teaching parents positive attention, problem solving, and effective communication skills via in vivo training with children.

Prolonged exposure

Overview

\section{Empirical support}

- Prolonged exposure for adolescents, a form of CBT, focuses primarily on psychoeducation and exposure, while also incorporating case management and relapse prevention.

- In an RCT of prolonged exposure versus time-limited dynamic therapy, both treatments resulted in symptom improvement, though effects were more pronounced in the prolonged exposure group [90, Class III]. 
Cognitive processing therapy

Overview

- Cognitive processing therapy involves psychoeducation, cognitive strategies, and documentation of thoughts and feelings at the time of the traumatic event via taped or written narratives.

Empirical support

- One RCT using a WLC illustrated promising findings [91, Class III].

Child-parent psychodynamic psychotherapy

Overview

Empirical support

- This treatment uses clinical illustrations and strategies to address various domains of functioning (e.g., play, sensorimotor, biological rhythms, fear, reckless behavior, aggression, parenting).

- One RCT comparing this intervention with case management demonstrated significant between-group differences [92, Class III].

\section{Compliance with Ethics Guidelines}

\section{Conflict of Interest}

Heather A. MacPherson declares that she has no conflict of interest.

Mary A. Fristad has served as a consultant for Wayne County Mental Health; has received grants from National Institute of Mental Health; has received payment for lectures from Ohio School Psychologists, Bert Nash Community Mental Health Center, American Academy of Child and Adolescent Psychiatry, University of Vermont, Brown School of Social Work, Washington University, American Board of Professional Psychology, American Psychological Association, and Association for Behavioral and Cognitive Therapies; and has received royalties from Guilford Press, American Psychiatric Publishing, and CFPSI Press.

Human and Animal Rights and Informed Consent

This article does not contain any studies with animal subjects performed by any of the authors.

\section{References and Recommended Reading}

Recently published papers of particular interest have been highlighted as:

- Of importance

$\bullet \quad$ Of major importance

1.• American Psychiatric Association. Diagnostic and statistical manual of mental disorders. 5th ed. Arlington, VA: American Psychiatric Publishing; 2013.

The $5^{\text {th }}$ edition of the Diagnostic and Statistical Manual of Mental Disorders offers updated diagnostic criteria for psychiatric disorders.

2. Merikangas KR, He JP, Burstein M, et al. Lifetime prevalence of mental disorders in U.S. adolescents: Results from the National Comorbidity Survey Replication-Adolescent Supplement (NCSA). J Am Acad Child Adolesc Psychiatry. 2010;49:980-9. 
3. Beesdo K, Knappe S, Pine DS. Anxiety and anxiety disorders in children and adolescents: developmental issues and implications for DSM-V. Psychiatr Clin North Am. 2009;32:483-524.

4. Costello EJ, Mustillo S, Erkanli A, et al. Prevalence and development of psychiatric disorders in childhood and adolescence. Arch Gen Psychiatry. 2003;60:837-44.

5. Van Meter AR, Moreira ALR, Youngstrom EA. Metaanalysis of epidemiologic studies of pediatric bipolar disorder. J Clin Psychiatry. 2011;72:1250-6.

This meta-analysis of epidemiological studies including both children and adolescents found an overall prevalence of 1.8 $\%$ for bipolar disorder. There was no significant difference in the mean rates between US and non-US studies, but the US studies had a wider range of rates. Highest prevalence rates came from studies using broad definitions of bipolar disorder (e.g., bipolar disorder not otherwise specified).

6. David-Ferdon C, Kaslow NJ. Evidence-based psychosocial treatments for child and adolescent depression. J Clin Child Adolesc Psychol. 2008;37:62104

7.• $\quad$ Fristad MA, MacPherson HA. Evidence-based psychosocial treatments for child and adolescent bipolar spectrum disorders. J Clin Child Adolesc Psychol. 2013 (in press).

This paper reviewed the evidence base for psychosocial treatment of pediatric bipolar spectrum disorders, using guidelines proposed by the Task Force on the Promotion and Dissemination of Psychological Procedures. No wellestablished or questionably efficacious treatments were identified. Family psychoeducation plus skill building was probably efficacious (i.e., multi-family psychoeducational psychotherapy, family-focused treatment). CBT was possibly efficacious. Dialectical behavior therapy and interpersonal and social rhythm therapy were experimental.

8. Silverman WK, Pina AA, Viswesvaran C. Evidencebased psychosocial treatments for phobic and anxiety disorders in children and adolescents. J Clin Child Adolesc Psychol. 2008;37:105-30.

9.• Freeman J, Garcia A, Frank H, et al. Evidence-base update for psychosocial treatments for pediatric obsessive-compulsive disorder. J Clin Child Adolesc Psychol. 2013 (in press).

This review provided an update on the evidence base for psychosocial treatment of pediatric OCD, using the Task Force on the Promotion and Dissemination of Psychological Procedures criteria. No well-established, experimental, or questionably efficacious treatments were identified. Individual and family-focused individual CBT were probably efficacious. Family-focused group CBT, non-familyfocused group CBT, and technology-based CBT were possibly efficacious.

10. Silverman WK, Ortiz CD, Viswesvaran C, et al. Evidence-based psychosocial treatments for children and adolescents exposed to traumatic events. J Clin Child Adolesc Psychol. 2008;37:156-83.
11. March J, Silva S, Petrycki S, et al. Fluoxetine, cognitive-behavioral therapy, and their combination for adolescents with depression: Treatment for Adolescents with Depression Study (TADS) randomized controlled trial. JAMA. 2004;292:807-20.

12. Kennard B, Silva S, Vitiello B, et al. Remission and residual symptoms after short-term treatment in the Treatment of Adolescents with Depression Study (TADS). J Am Acad Child Adolesc Psychiatry. 2006;45:1404-11.

13. Brent D, Emslie G, Clarke G, et al. Switching to another SSRI or to venlafaxine with or without cognitive behavioral therapy for adolescents with SSRIresistant depression: the TORDIA randomized controlled trial. JAMA. 2008;299:901-13.

14. Melvin GA, Tonge BJ, King NJ, et al. A comparison of cognitive-behavioral therapy, sertraline, and their combination for adolescent depression. J Am Acad Child Adolesc Psychiatry. 2006;45:1151-61.

15. Goodyer I, Dubicka B, Wilkinson P, et al. Selective serotonin reuptake inhibitors (SSRIs) and routine specialist care with and without cognitive behaviour therapy in adolescents with major depression: randomised controlled trial. BMJ. 2007;335:142.

16. Brent DA, Holder D, Kolko D, et al. A clinical psychotherapy trial for adolescent depression comparing cognitive, family, and supportive therapy. Arch Gen Psychiatry. 1997;54:877-85.

17. Clarke GN, Rohde P, Lewinsohn PM, et al. Cognitive-behavioral treatment of adolescent depression: Efficacy of acute group treatment and booster sessions. J Am Acad Child Adolesc Psychiatry. 1999;38:272-9.

18. Rohde P, Clarke GN, Mace DE, et al. An efficacy/effectiveness study of cognitive behavioral treatment for adolescents with and without comorbid major depression and conduct disorder. J Am Acad Child Adolesc Psychiatry. 2004;43:660-8.

19. Wood A, Harrington R, Moore A. Controlled trial of a brief cognitive-behavioural intervention in adolescent patients with depressive disorders. J Child Psychol Psychiatry. 1996;37:737-46.

20. Weisz JR, Southam-Gerow MA, Gordis EB, et al. Cognitive-behavioral therapy versus usual clinical care for youth depression: an initial test of transportability to community clinics and clinicians. J Consult Clin Psychol. 2009;77:383-96.

21. Nelson EL, Barnard M, Cain S. Treating childhood depression over videoconferencing. Telemed J E Health. 2003;9:49-55.

22. Vostanis P, Feehan C, Grattan E, Bickerton WL. A randomised controlled out-patient trial of cognitivebehavioural treatment for children and adolescents with depression: 9-month follow-up. J Affect Disord. 1996;40:105-16. 
23. Kovacs M, Sherrill J, George CJ, et al. Contextual emotion-regulation therapy for childhood depression: description and pilot testing of a new intervention. J Am Acad Child Adolesc Psychiatry. 2006;45:892-903.

24. Tompson MC, Pierre CB, Haber FM, et al. Familyfocused treatment for childhood-onset depressive disorders: results of an open trial. Clin Child Psychol Psychiatry. 2007;12:403-20.

25.• Weisz JR, Chorpita BF, Palinkas LA, et al. Testing standard and modular designs for psychotherapy treating depression, anxiety, and conduct problems in youth: a randomized effectiveness trial. Arch Gen Psychiatry. 2012;69:274-82.

This effectiveness RCT used a mixed sample of school-aged children presenting with depression, anxiety, and/or conduct problems and demonstrated superiority of a modular approach (flexible application of CBT protocols) over usual care and standard evidence-based treatments (separate, disorder-specific protocols). This study is significant, given the limited amount of effectiveness trials with children to date, and also suggests that flexible delivery of evidence-based treatments may be more impactful for youth in community settings.

26. Mufson L, Dorta KP, Wickramaratne P, et al. A randomized effectiveness trial of interpersonal psychotherapy for depressed adolescents. Arch Gen Psychiatry. 2004;61:577-84.

27. Mufson L, Weissman MM, Moreau D, et al. Efficacy of interpersonal psychotherapy for depressed adolescents. Arch Gen Psychiatry. 1999;56:573-9.

28. Rossello J, Bernal G. The efficacy of cognitive-behavioral and interpersonal treatments for depression in Puerto Rican adolescents. J Consult Clin Psychol. 1999; 67:734-45.

29. Rossello J, Bernal G, Rivera-Medina C. Individual and group CBT and IPT for Puerto Rican adolescents with depressive symptoms. Cultur Divers Ethnic Minor Psychol. 2008;14:234-45.

30. Dietz LJ, Mufson L, Irvine H, et al. Family-based interpersonal psychotherapy for depressed preadolescents: an open-treatment trial. Early Interv Psychiatry. 2008;2:154-61.

31. Diamond GS, Reis BF, Diamond GM, et al. Attachment based family therapy for depressed adolescents: a treatment development study. J Am Acad Child Adolesc Psychiatry. 2002;41:1190-6.

32. Israel P, Diamond GS. Feasibility of attachment based family therapy for depressed clinic-referred Norwegian adolescents. Clin Child Psychol Psychiatry. 2012;18:334-50.

33. Trowell J, Joffe I, Campbell JC, et al. Childhood depression: a place for psychotherapy. An outcome study comparing individual psychodynamic psychotherapy and family therapy. Eur Child Adolesc Psychiatry. 2007;16:157-67.
34. Luby J, Lenze S, Tillman R. A novel early intervention for preschool depression: findings from a pilot randomized controlled trial. J Child Psychol Psychiatry. 2012;53:313-22.

In the first RCT of a psychosocial intervention for preschoolers diagnosed with major depressive disorder, PCITED was superior to psychoeducation control in improving executive functioning and emotion recognition skills. Both groups also showed improvement in several other domains, though PCIT-ED showed significance in a greater number of areas.

35. Dopp RR, Mooney AJ, Armitage R, King C. Exercise for adolescents with depressive disorders: a feasibility study. Depress Res Treat. 2012;2012:257472.

36. Fristad MA, Verducci JS, Walters K, Young ME. Impact of multifamily psychoeducational psychotherapy in treating children aged 8 to 12 years with mood disorders. Arch Gen Psychiatry. 2009;66:1013-20.

37. Miklowitz DJ, Axelson DA, Birmaher B, et al. Familyfocused treatment for adolescents with bipolar disorder: results of a 2-year randomized trial. Arch Gen Psychiatry. 2008;65:1053-61.

38.• Miklowitz DJ, Schneck CD, Singh, et al. Early intervention for symptomatic youth at risk for bipolar disorder: a randomized trial of family-focused therapy. J Am Acad Child Adolesc Psychiatry. 2013;52:121-31.

This is one of three rigorously conducted RCTs that examined efficacy of a psychosocial intervention for youth at high risk for developing bipolar disorders (FFT-HR). Compared with family education control+ pharmacotherapy, FFT-HR+ pharmacotherapy resulted in more rapid recovery from initial mood symptoms, more weeks in remission, and a more favorable trajectory of manic symptoms over one year. The magnitude of the treatment effect was greater among youth with families exhibiting high (versus low) expressed emotion.

39. Boylan K, MacPherson HA, Fristad MA. Examination of disruptive behavior outcomes and moderation in a randomized psychotherapy trial for mood disorders. J Am Acad Child Adolesc Psychiatry. 2013;52:699-708.

This secondary analysis of the multi-family psychoeducational psychotherapy (MF-PEP) +TAU versus WLC+TAU RCT for treatment of pediatric mood disorders evaluated disruptive behavior outcomes and moderation. Greater degree of disruptive behaviors was associated with worse mood symptoms and impairment. Between-group analyses examining outcome of disruptive behaviors were nonsignificant. Within-group analyses and between-group effect sizes suggested that MF-PEP was associated with decreases in attention-deficit/hyperactivity disorder, oppositional defiant disorder, and overall disruptive behavior symptoms, but not conduct disorder symptoms. Baseline severity of disruptive behaviors did not affect treatment response of mood symptoms to MF-PEP. 
40. Pavuluri MN, Graczyk PA, Henry DB, et al. Childand family-focused cognitive-behavioral therapy for pediatric bipolar disorder: development and preliminary results. J Am Acad Child Adolesc Psychiatry. 2004;43:528-37.

41. West AE, Jacobs RH, Westerholm R, et al. Child and family-focused cognitive-behavioral therapy for pediatric bipolar disorder: pilot study of group treatment format. J Can Acad Child Adolesc Psychiatry. 2009;18:239-46.

42. Feeny NC, Danielson CK, Schwartz L, et al. Cognitivebehavioral therapy for bipolar disorders in adolescents: a pilot study. Bipolar Disord. 2006;8:508-15.

43. Goldstein TR, Axelson DA, Birmaher B, Brent DA. Dialectical behavior therapy for adolescents with bipolar disorder: a 1-year open trial. J Am Acad Child Adolesc Psychiatry. 2007;46:820-30.

44. Hlastala SA, Kotler JS, McClellan JM, McCauley EA. Interpersonal and social rhythm therapy for adolescents with bipolar disorder: treatment development and results from an open trial. Depress Anxiety. 2010;27:457-64.

45. Walkup JT, Albano AM, Piacentini J, et al. Cognitive behavioral therapy, sertraline, or a combination in childhood anxiety. N Engl J Med. 2008;359:2753-66.

46. Barrett PM. Evaluation of cognitive-behavioral group treatments for childhood anxiety disorders. J Clin Child Psychol. 1998;27:459-68.

47. Cartwright-Hatton S, McNally D, Field AP, et al. A new parenting-based group intervention for young anxious children: results of a randomized controlled trial. J Am Acad Child Adolesc Psychiatry.

2011;50:242-51.

Given the limited amount of research devoted to treatment of anxiety disorders in young children, this RCT investigated and demonstrated efficacy of a parent-only, group CBT treatment, when compared with WLC, for children 9 years and younger. Results were maintained at 12-month follow-up.

48. Hirshfeld-Becker DR, Masek B, Henin A, et al. Cognitive Behavioral therapy for 4- to 7-year-old children with anxiety disorders: a randomized clinical trial. J Consult Clin Psychol. 2010;78:498-510.

49. Hudson JL, Rapee RM, Deveney C, et al. Cognitivebehavioral treatment versus an active control for children and adolescents with anxiety disorders: a randomized trial. J Am Acad Child Adolesc Psychiatry. 2009;48:533-44.

50. Kendall PC, Hudson JL, Gosch E, et al. Cognitivebehavioral therapy for anxiety disordered youth: a randomized clinical trial evaluating child and family modalities. J Consult Clin Psychol. 2008;76:282-97.

51. Schneider S, Blatter-Meunier S, Herren J, In-Albon C. The efficacy of a family-based cognitive-behavioral treatment for separation anxiety disorder in children aged 8-13. A randomized comparison with a general anxiety program. J Consult Clin Psychol.

2012;81:932-40.
This RCT examined the efficacy of a CBT-based, family-involved, disorder-specific treatment for children with separation anxiety versus a general, child-focused CBT program. Both treatments resulted in significant improvement in anxiety symptoms and parental dysfunctional beliefs. Thus, parental component and disorder-specific interventions may not offer added benefit beyond general, child-based CBT treatments.

52. Southam-Gerow MA, Weisz JR, Chu BC, et al. Does cognitive behavioral therapy for youth anxiety outperform usual care in community clinics? An initial effectiveness test. J Am Acad Child Adolesc Psychiatry. 2010;49:1043-52.

53. Gallagher HM, Rabian BA, McCloskey MS. A brief group cognitive behavioral intervention for social phobia in childhood. J Anxiety Disord. 2004;18:459-79.

54. Masia Warner C, Fisher PH, Shrout PE, et al. Treating adolescents with social anxiety disorder in school: an attention control trial. J Child Psychol Psychiatry. 2007;48:676-86.

55. Wuthrich VM, Rapee RM, Cunningham MJ, et al. A randomized controlled trial of the Cool Teens CD-ROM computerized program for adolescent anxiety. J Am Acad Child Adolesc Psychiatry. 2012;51:261-70.

56. Spence SH, Donovan CL, March S, et al. A randomized controlled trial of online versus clinic-based CBT for adolescent anxiety. J Consult Clin Psychol. 2011;79:629-42.

In this RCT, online and clinic delivery of CBT with clinically anxious adolescents produced significant and comparable improvements in anxiety symptoms through 12-month follow-up. Both were also superior to WLC. Treatment credibility and satisfaction were high among the two CBT formats. Thus, this study offers avenues for transportability and dissemination of CBT via multiple formats.

57. Khanna MS, Kendall PC. Computer-assisted cognitive behavioral therapy for child anxiety: results of a randomized clinical trial. J Consult Clin Psychol. 2010;78:737-45.

58. Beidel DC, Turner SM, Morris TL. Behavioral treatment of childhood social phobia. J Consult Clin Psychol. 2000;68:1072-80.

59. Beidel DC, Turner SM, Sallee FR, et al. SET-C versus fluoxetine in the treatment of childhood social phobia. J Am Acad Child Adolesc Psychiatry. 2007;46:1622-32.

$60 . \bullet$ Eldar S, Apter A, Lotan D, et al. Attention bias modification treatment for pediatric anxiety disorders: a randomized controlled trial. Am J Psychiatry. 2012;169:213-20.

In this RCT, the novel intervention ABMT (learning to shift attention away from threat stimuli via computer training tasks) resulted in significant reduction in childhood anxiety symptoms compared to placebo attention training with threat and neutral stimuli. Importantly, this was the first RCT of ABMT in anxious children. 
61. Waters AM, Pittaway M, Mogg K, et al. Attention training towards positive stimuli in clinically anxious children. Dev Cogn Neurosci. 2013;4:77-84.

62. Comer JS, Puliafico AC, Aschenbrand SG, et al. A pilot feasibility evaluation of the CALM Program for anxiety disorders in early childhood. J Anxiety Disord. 2012;26:40-9.

63. Silverman WK, Kurtines WM, Ginsburg GS, et al. Contingency management, self-control, and education support in the treatment of childhood phobic disorders: a randomized clinical trial. J Consult Clin Psychol. 1999;67:675-87.

64. Ollendick TH, Ost L, Reuterskiöld L, et al. One-session treatment of specific phobias in youth: a randomized clinical trial in the United States and Sweden. J Consul Clin Psychol. 2009;77:504-16.

65. Pincus DB, May JE, Whitton SW, et al. Cognitivebehavioral treatment of panic disorder in adolescence. J Clin Child Adolesc Psychol. 2010;39:63849.

66. Pediatric OCD Treatment Study Team. Cognitivebehavior therapy, sertraline, and their combination for children and adolescents with obsessive-compulsive disorder: the Pediatric OCD Treatment Study (POTS) randomized controlled trial. JAMA. 2004;292:1969-76.

67.• Franklin ME, Sapyta J, Freeman JB, et al. Cognitive behavior therapy augmentation of pharmacotherapy in pediatric obsessive-compulsive disorder: the $\mathrm{Pe}$ diatric OCD Treatment Study II (POTS II) randomized controlled trial. JAMA. 2011;306:1224-32.

This is the second rigorously conducted RCT to examine efficacy of individual CBT + pharmacotherapy in treatment of pediatric OCD. Findings indicated superiority of CBT+pharmacotherapy over pharmacotherapy alone and pharmacotherapy+instructions in CBT for youth with OCD. Thus, results underlie the importance of thorough CBT, in addition to medication, in the treatment of pediatric OCD.

68. Farrell LJ, Waters AM, Boschen MJ, Hattingh L. Difficult-to-treat pediatric obsessive-compulsive disorder: feasibility and preliminary results of a randomized pilot trial of D-cycloserine-augmented behavior therapy. Depress Anxiety. 2013;30:723-31. This is one of two RCTs demonstrating superiority of individual $\mathrm{CBT}+\mathrm{D}$-cycloserine versus $\mathrm{CBT}+$ placebo in treatment of youth with OCD. D-cycloserine is hypothesized to aid in extinction learning during exposure and response prevention exercises, and has shown recent promising results in both adult and child anxiety treatment outcome research.

69. Storch EA, Murphy TK, Goodman WK, et al. A preliminary study of D-cycloserine augmentation of cognitive-behavioral therapy in pediatric obsessivecompulsive disorder. Biol Psychiatry. 2010;68:10736.

70. Barrett P, Healy-Farrell L, March JS. Cognitive behavioral family treatment of childhood obsessivecompulsive disorder: a controlled trial. J Am Acad Child Adolesc Psychiatry. 2004;43:46-62.
71. Freeman JB, Garcia AM, Coyne L, et al. Early childhood OCD: preliminary findings from a familybased cognitive-behavioral approach. J Am Acad Child Adolesc Psychiatry. 2008;47:593-602.

72. Piacentini J, Bergman RL, Chang S, et al. Controlled comparison of family cognitive behavioral therapy and psychoeducation-relaxation training for child obsessive-compulsive disorder. J Am Acad Child Adolesc Psychiatry. 2011;50:1149-61.

73. Olino TM, Gillo S, Rowe D, et al. Evidence for successful implementation of exposure and response prevention in a naturalistic group format for pediatric OCD. Depress Anxiety. 2011;28:342-8.

74. Sochting I, Third B. Behavioral group treatment for obsessive-compulsive disorder in adolescence: a pilot study. Int J Group Psychother. 2011;61:84-97.

75. Storch EA, Caporino NE, Morgan JR, et al. Preliminary investigation of web-camera delivered cognitivebehavioral therapy for youth with obsessive compulsive disorder. Psychiatry Res. 2011;189:407-12.

76. Cohen JA, Deblinger E, Mannarino AP, Steer RA. A multisite randomized controlled study of sexually abused, multiply traumatized children with PTSD: initial treatment outcome. J Am Acad Child Adolesc Psychiatry. 2004;43:393-402.

77.• Cohen JA, Mannarino AP, Iyengar S. Community treatment of posttraumatic stress disorder for children exposed to intimate partner violence. Arch Pediatr Adolesc Med. 2011;165:16-21.

Given the substantial amount of evidence supporting the efficacy of TF-CBT, this study aimed to examine effectiveness of this intervention when delivered in the community. Compared with usual care, community-delivered TF-CBT resulted in significantly greater improvements in anxiety and PTSD symptoms, thus supporting the transport of TF-CBT to practice settings.

78. Kolko DJ. Individual cognitive behavioral treatment and family treatment and family therapy for physically abused children and their offending parents: a comparison of clinical outcomes. Child Maltreatment. 1996;1:322-42.

79. Scheeringa MS, Weems CF, Cohen JA, et al. Traumafocused cognitive-behavioral therapy for posttraumatic stress disorder in three to six year-old children: a randomized clinical trial. J Child Psychol Psychiatry. 2011;52:853-560.

This study examined use of TF-CBT with 3- to 6-year-olds with heterogeneous trauma histories, given the dearth of research on effective trauma-focused treatments for preschoolers, most of which have focused on childhood sexual abuse. Relative to WLC, TF-CBT was effective in reducing symptoms of PTSD, and there was some evidence for improvement in comorbid disorders.

80. Cohen JA, Mannarino AP, Perel JM, Staron V. A pilot randomized controlled trial of combined traumafocused CBT and sertraline for childhood PTSD symptoms. J Am Acad Child Adolesc Psychiatry. 2007;46:811-9. 
81. Nixon RD, Sterk J, Pearce A. A randomized trial of cognitive behaviour therapy and cognitive therapy for children with posttraumatic stress disorder following single-incident trauma. J Abnorm Child Psychol. 2012;40:327-37.

82. Smith P, Yule W, Perrin S, et al. Cognitive-behavioral therapy for PTSD in children and adolescents: a preliminary randomized controlled trial. J Am Acad Child Adolesc Psychiatry. 2007;46:1051-61.

83. Catani C, Kohiladevy M, Ruf M, et al. Treating children traumatized by war and tsunami: a comparison between exposure therapy and meditation-relaxation in North-East Sri Lanka. BMC Psychiatry. 2009;9:22.

84. Jaycox LH, Cohen JA, Mannarino AP, et al. Children's mental health care following hurricane Katrina: a field trial of trauma-focused psychotherapies. J Trauma Stress. 2010;23:223-31.

85. Layne CM, Saltzman WR, Poppleton L, et al. Effectiveness of a school-based psychotherapy program for war-exposed adolescents: a randomized controlled trial. J Am Acad Child Adolesc Psychiatry. 2008;47:1048-62.

86. Jaberghaderi N, Greenwald R, Rubin A, et al. A comparison of CBT and EMDR for sexually abused Iranian girls. Clin Psychol Psychother. 2004;11:358-68.

87. De Roos C, Greenwald R, den Hollander-Gijsman M, et al. A randomized comparison of cognitive behavioural therapy (CBT) and eye movement desensitization and reprocessing (EMDR) in disaster-exposed children. Eur J Psychotraumatol. 2011;2.

88. Thomas R, Zimmer-Gembeck MJ. Accumulating evidence for parent-child interaction therapy in the prevention of child maltreatment. Child Dev. 2011;82:177-92.

89.• Thomas R, Zimmer-Gembeck MJ. Parent-child interaction therapy: an evidence-based treatment for child maltreatment. Child Maltreat. 2012;17:253-66.

In the second RCT of PCIT for families at risk for or with a history of maltreatment, a relatively brief version of PCIT (12 sessions) resulted in fewer externalizing and internalizing behaviors, decreased stress, more positive verbalizations, and increased maternal sensitivity, relative to WLC.

90. Gilboa-Schechtman E, Foa EB, Shafran N, Aderka IM. Prolonged exposure versus dynamic therapy for adolescent PTSD: a pilot randomized controlled trial. J Am Acad Child Adolesc Psychiatry. 2010;49:1034-42.

91. Ahrens J, Rexford L. Cognitive processing therapy for incarcerated adolescents with PTSD. J Aggression Maltreat Trauma. 2002;6:201-16.

92. Lieberman AF, Van Horn P, Ippen CG. Toward evidence-based treatment: child-parent psychotherapy with preschoolers exposed to marital violence. J Am Acad Child Adolesc Psychiatry. 2005;44:1241-8. 